Research Article

\title{
Cosine Similarity Measure of Complex Fuzzy Sets and Robustness of Complex Fuzzy Connectives
}

\author{
Wenping Guo, ${ }^{1}$ Lvqing $\mathrm{Bi}^{2}{ }^{2} \mathrm{Bo} \mathrm{Hu}^{3}$ and Songsong Dai ${ }^{1}{ }^{1}$ \\ ${ }^{1}$ School of Electronics and Information Engineering, Taizhou University, Taizhou 318000, China \\ ${ }^{2}$ School of Electronics and Communication Engineering, \\ Guangxi Colleges and Universities Key Laboratory of Complex System Optimization and Big Data Processing, \\ Yulin Normal University, Yulin 537000, China \\ ${ }^{3}$ School of Mechanical and Electrical Engineering, Guizhou Normal University, Guiyang 550025, China
}

Correspondence should be addressed to Songsong Dai; ssdai@stu.xmu.edu.cn

Received 18 April 2020; Accepted 3 July 2020; Published 22 July 2020

Academic Editor: Anna Vila

Copyright (C) 2020 Wenping Guo et al. This is an open access article distributed under the Creative Commons Attribution License, which permits unrestricted use, distribution, and reproduction in any medium, provided the original work is properly cited.

Complex fuzzy set (CFS), as a generalization of fuzzy set (FS), is characterized by complex-valued membership degrees. By considering the complex-valued membership degree as a vector in the complex unit disk, we introduce the cosine similarity measures between CFSs. Then, we investigate some invariance properties of the cosine similarity measure. Finally, the cosine similarity measure is applied to measure the robustness of complex fuzzy connectives and complex fuzzy inference.

\section{Introduction}

Similarity measure is an important tool in fuzzy mathematics. It has been successfully applied to many fields [1-3]. Cosine similarity measure is a special type of similarity measure which is viewed as the cosine of the angle between two vectors $[4,5]$. In order to define the cosine similarity measure for FSs, membership degree in FSs is used by the vector representation. By this method, some cosine similarity measures are proposed for intuitionistic fuzzy sets and applied to pattern recognition and medical diagnosis [6,7]. And some cosine similarity measures for interval-valued intuitionistic fuzzy sets [8], Pythagorean fuzzy sets [9], picture fuzzy sets [10], vague sets [11], hesitant fuzzy set [12], and neutrosophic sets [13] have been proposed. However, there is no cosine similarity measures between CFSs. As we have known, in many studies, CFSs [14, 15] are viewed as sets of vectors of the complex unit disk. In [16], Dick considered complex fuzzy logic as a logic of vectors and introduced a feature called rotational invariance for complex fuzzy operations. Later, this feature is examined for some measures and operations of CFSs [17-19] and intervalvalued CFSs [20-25]. Ramot et al. [15] considered complex fuzzy aggregation as a vector aggregation on CFSs. $\mathrm{Hu}$ et al. [26-28] introduced the orthogonality and parallelity relations for CFSs based on the geometrical relations of the vectors of complex-valued membership degrees. So, it is natural to define cosine similarity measures between CFSs when they are considered as sets of vectors in the complex unit disk.

The robustness of fuzzy inference has become a particular topic in the research area of fuzzy inference [29-39]. In the research of robustness of complex fuzzy inference, one fundamental problem is how to measure the perturbation of CFSs. Distance and similarity measures between CFSs play an important role in measuring the robustness of complex fuzzy inference methods. Zhang et al. [40] proposed a notion of $\delta$-equality of CFSs. Alkouri et al. [41] and $\mathrm{Hu}$ et al. [42] introduced several distance measures for CFSs. As we know, CFSs is defined by a phase term and an amplitude term. These distances are the average or maximin of the difference between the amplitude terms and the difference between the phase terms. As mentioned in [14], the phase term of CFS is the key element that distinguishes CFSs from other extensions of fuzzy sets. We can study the robustness of complex fuzzy operators and complex fuzzy inference methods based on above distances. Zhang et al. [40] studied the robustness of complex fuzzy operations based on the $\delta$-equality of CFSs. However, it cannot provide an effective method for measuring the robustness of the phase term of complex fuzzy operators and complex fuzzy inference. Having 
this in mind, we propose the cosine similarity measure for CFSs which just relies on the phase term of CFS. Then, we study the robustness of the phase term of complex fuzzy connectives and complex fuzzy inference based on this similarity of CFSs.

In this paper, we focus on the cosine similarity measure between CFSs. First we review some necessary concepts of CFSs in Section 2. In Section 3, we propose the cosine similarity measure between CFSs and discuss its invariance properties. In Section 4, we compare our measure with other existing measures. In Section 5, the cosine similarity measures are applied to measure the robustness of complex fuzzy connectives and complex fuzzy inference. Finally, conclusions are given in Section 6.

\section{Preliminaries}

In this paper, our discussion is based on CFS theory. Some basic concepts of CFSs are recalled below, whereas others are given in [14-16].

Let $O$ be the set of complex numbers on complex unit disk, i.e., $O=\{\alpha \in \mathbb{C}|| \alpha \mid \leq 1\}$. Let $X$ be a fixed universe; a mapping $S: X \longrightarrow D$ is called a CFS in $X$, whose its membership degree $\mu_{S}(x)$ is

$$
r_{S}(x) \cdot e^{j v_{S}(x)}
$$

where $j=\sqrt{-1}, r_{S}(x) \in[0,1]$ is the amplitude part, and $\nu_{S}(x) \in \mathbb{R}$ is the phase part.

Three complex fuzzy complement operations are defined by Ramot et al. [14] as follows:

$$
\begin{aligned}
\left(\Gamma_{1} S\right)(x) & =\left(1-r_{S}(x)\right) \cdot e^{j\left(-v_{S}(x)\right)}, \\
\left(\Gamma_{2} S\right)(x) & =\left(1-r_{S}(x)\right) \cdot e^{j\left(v_{S}(x)\right)}, \\
\left(\Gamma_{3} S\right)(x) & =\left(1-r_{S}(x)\right) \cdot e^{j\left(v_{S}(x)+\pi\right)} .
\end{aligned}
$$

Let $A$ and $B$ be two CFSs; intersection and union of $A$ and $B$ are defined by Ramot et al. [14] as follows:

$$
\begin{aligned}
& (A \cup B)(x)=\left(r_{A}(x) * r_{B}(x)\right) \cdot e^{j v_{A \otimes B}(x),} \\
& (A \cap B)(x)=\left(r_{A}(x) \star r_{B}(x)\right) \cdot e^{j v_{A \otimes B}(x)},
\end{aligned}
$$

where $*$ and $\star$ represent a $\mathrm{t}$-conorm and a $\mathrm{t}$-norm, respectively.

Some commonly used functions of $v_{A \otimes B}$ are defined as follows (see [14]):

$$
\begin{aligned}
& v_{A+B}=v_{A}+v_{B}, \\
& v_{A \vee B}=\max \left(v_{A}, v_{B}\right), \\
& v_{A \wedge B}=\min \left(v_{A}, v_{B}\right), \\
& v_{A-B}=v_{A}-v_{B} .
\end{aligned}
$$

Rotation and reflection operations of CFSs are defined by Ramot et al. [14] as follows:

(i) Rotation of a CFS $S$ :

$$
\operatorname{Rot}_{\theta}(S)(x)=r_{S}(x) \cdot e^{j v_{S}(x)+\theta},
$$

where $\theta \in \mathbb{R}$.

(ii) Reflection of a CFS S:

$$
\operatorname{Ref}(S)(x)=r_{S}(x) \cdot e^{-j v_{S}(x)} .
$$

A dependency relation of CFSs is defined by $\mathrm{Hu}$ et al. [26] as follows. Two CFSs $A$ and $B$ are said to be orthogonal, denoted by $A \perp B$, if

$$
<\mu_{A}(x), \mu_{B}(x)>=0,
$$

for any $x \in X$, where $<\mu_{A}(x), \mu_{B}(x)>$ is the inner product of complex numbers $\mu_{A}(x), \mu_{B}(x) \in O$.

\section{Cosine Similarity Measures for CFSs}

In this section, we propose a cosine similarity measure between CFSs.

Let $A$ and $B$ be two CFSs in $X=\left\{x_{1}, x_{2}, \ldots, x_{n}\right\}$; a cosine similarity measure between two CFSs $A$ and $B$ is defined as follows:

$$
C_{\mathrm{CFS}}(A, B)=\bigvee_{i=1}^{n} \frac{<\mu_{A}(x), \mu_{B}(x)>}{\left|\mu_{A}(x)\right| \cdot\left|\mu_{B}(x)\right|}
$$

Obviously, assume that $\theta_{i}(i=1,2, \ldots, n)$ is the angle of two vector $\mu_{A}\left(x_{i}\right)$ and $\mu_{B}\left(x_{i}\right)$; then,

$$
C_{\mathrm{CFS}}(A, B)=\bigvee_{i=1}^{n} \cos \left(\theta_{i}\right)
$$

Theorem 1. Suppose that $A, B$ are two CFSs in $X$; the cosine similarity measure $C_{C F S}(A, B)$ of $A$ and $B$ satisfies the following properties:

(1) $-1 \leq C_{C F S}(A, B) \leq 1$

(2) $C_{C F S}(A, B)=C_{C F S}(B, A)$

(3) $C_{C F S}(A, A)=1$

(4) $C_{C F S}(A, B)=-1$ if $A=-B$, i.e., $\mu_{A}(x)=-\mu_{B}(x)$ for all $x \in X$

(5) $C_{C F S}(A, B)=0$ if $A \perp B$

Proof

(1) Since $-1 \leq \cos \left(\theta_{i}\right) \leq 1$, then $-1 \leq \mathrm{V}_{i=1}^{n} \cos \left(\theta_{i}\right) \leq 1$.

(2) It is obvious from $<\mu_{A}(x), \mu_{B}(x)>=<\mu_{B}(x)$, $\mu_{A}(x)>$ and $\left|\mu_{A}(x)\right| \cdot\left|\mu_{B}(x)\right|=\left|\mu_{B}(x)\right| \cdot\left|\mu_{A}(x)\right|$ for all $x \in X$.

(3) It is obvious from $\left|\mu_{A}(x)\right| \cdot\left|\mu_{A}(x)\right|=<\mu_{A}(x)$, $\mu_{A}(x)>$ for all $x \in X$.

(4) For any $x \in X$, when $\mu_{A}(x)=-\mu_{B}(x),<\mu_{A}(x)$, $\mu_{A}(x)>=-\left|\mu_{A}(x)\right| \cdot\left|\mu_{A}(x)\right|$. So, we have $C_{\mathrm{CFS}}(A, B)=-1$.

(5) When $A \perp B$, then the angle of two vector $\mu_{A}(x)$ and $\mu_{B}(x)$ is $\pi / 2$ for all $x \in X$. So, we have $C_{\mathrm{CFS}}(A, B)=0$ from $\cos (\pi / 2)=0$.

If we define the distance measure of CFSs by $D_{\mathrm{CFS}}(A, B)=\arccos \left(C_{\mathrm{CFS}}(A, B)\right)$, then it satisfies the following properties. 
Theorem 2. Suppose that $A, B$ are two CFSs in $X$; the distance measure $D_{C F S}(A, B)$ of $A$ and $B$ satisfies the following properties:

(1) $0 \leq D_{C F S}(A, B) \leq \pi$.

(2) $D_{C F S}(A, B)=D_{C F S}(B, A)$

(3) $D_{C F S}(A, A)=0$

Proof. Equations (1)-(3) are obvious from $-1 \leq C_{\mathrm{CFS}}(A, B)$ $\leq 1, \quad C_{\mathrm{CFS}}(A, B)=C_{\mathrm{CFS}}(B, A), \quad$ and $\quad C_{\mathrm{CFS}}(A, A)=1$, respectively.

The cosine similarity measure for CFSs is reflectionally invariant and rotationally invariant.

Theorem 3. Suppose that $A, B$ are two CFSs in $X$; the cosine similarity measure $C_{C F S}(A, B)$ of $A$ and $B$ is reflectionally invariant and rotationally invariant, i.e.,

$$
\begin{gathered}
C_{\mathrm{CFS}}(\operatorname{Ref}(A), \operatorname{Ref}(B))=C_{\mathrm{CFS}}(A, B), \\
C_{\mathrm{CFS}}\left(\operatorname{Rot}_{\theta}(A), \operatorname{Rot}_{\theta}(B)\right)=C_{\mathrm{CFS}}(A, B),
\end{gathered}
$$

for any $\theta$.

Proof. For any $x_{i} \in X$, assume that the angle of two vector $\mu_{A}\left(x_{i}\right)$ and $\mu_{B}\left(x_{i}\right)$ is $\theta_{i}$; then, the angle of two vector $\mu_{\operatorname{Ref}(A)}\left(x_{i}\right)$ and $\mu_{\operatorname{Ref}(B)}\left(x_{i}\right)$ is also $\theta_{i}$. Thus, $C_{\mathrm{CFS}}(\operatorname{Ref}(A), \operatorname{Ref}(B))=C_{\mathrm{CFS}}(A, B)$. Similarly, we have $C_{\mathrm{CFS}}\left(\operatorname{Rot}_{\theta}(A), \operatorname{Rot}_{\theta}(B)\right)=C_{\mathrm{CFS}}(A, B)$ for any $\theta$.

Theorem 4. Suppose that $A, B$ are two CFSs in $X$; the distance measure $D_{C F S}(A, B)$ of $A$ and $B$ is reflectionally invariant and rotationally invariant, i.e.,

$$
\begin{gathered}
D_{\mathrm{CFS}}(\operatorname{Ref}(A), \operatorname{Ref}(B))=D_{\mathrm{CFS}}(A, B), \\
D_{\mathrm{CFS}}\left(\operatorname{Rot}_{\theta}(A), \operatorname{Rot}_{\theta}(B)\right)=D_{\mathrm{CFS}}(A, B),
\end{gathered}
$$

for any $\theta$.

Proof. Trivial from Theorem 3.

Moreover, the cosine similarity measure for CFSs is ratio scale invariant.

Theorem 5. Suppose that $A, B$ are two CFSs in $X$; the cosine similarity measure $C_{C F S}(A, B)$ of $A$ and $B$ is ratio scale invariant, i.e.,

$$
C_{\mathrm{CFS}}(t A, t B)=C_{\mathrm{CFS}}(A, B) \text {, }
$$

for any $t>0$ such that $t A(x), t B(x) \in O$, where $\mu_{t A}(x)=$ $\left(t \cdot r_{A}(x)\right) \cdot e^{j v_{A}(x)}$ for all $x \in X$.

Proof. It can be easily obtained from the fact that the angle of two vector $\mu_{A}(x)$ and $\mu_{B}(x)$ is same to the angle of two vector $\mu_{t A}(x)$ and $\mu_{t B}(x)$ for any $x \in X$.

Theorem 6. Suppose that $A, B$ are two CFSs in $X$; the distance measure $D_{C F S}(A, B)$ of $A$ and $B$ is ratio scale invariant, i.e.,

$$
D_{\mathrm{CFS}}(t A, t B)=D_{\mathrm{CFS}}(A, B)
$$

for any $t>0$ such that $t A(x), t B(x) \in O$.

Proof. Trivial from Theorem 5.

\section{Comparisons of Distance Measures for CFSs}

Since there are no other similarity measures of CFSs, in this section, we make a comparison between the proposed distance measure $D_{\mathrm{CFS}}(A, B)=\arccos \left(C_{\mathrm{CFS}}(A, B)\right)$ and other existing distance measures in CFSs [40-42]. Let $A$ and $B$ be two CFSs in $X=\left\{x_{1}, x_{2}, \ldots, x_{n}\right\}$; some existing distance measures between $A$ and $B$ are listed as follows:

$$
\begin{aligned}
D_{Z}(A, B) & =\max \left(\sup _{x \in X}\left|r_{A}(x)-r_{B}(x)\right|, \sup _{x \in X} \frac{\left|v_{A}(x)-v_{B}(x)\right|}{2 \pi}\right), \\
D_{H}(A, B) & =\frac{1}{2} \sum_{i=1}^{n}\left(\left|r_{A}\left(x_{i}\right)-r_{B}\left(x_{i}\right)\right|+\frac{\left|v_{A}\left(x_{i}\right)-v_{B}\left(x_{i}\right)\right|}{2 \pi}\right), \\
D_{n H}(A, B) & =\frac{1}{2 n} \sum_{i=1}^{n}\left(\left|r_{A}\left(x_{i}\right)-r_{B}\left(x_{i}\right)\right|+\frac{\left|v_{A}\left(x_{i}\right)-v_{B}\left(x_{i}\right)\right|}{2 \pi}\right), \\
D_{E}(A, B) & =\left[\frac{1}{2}\left(\sum_{i=1}^{n}\left(\left(r_{A}\left(x_{i}\right)-r_{B}\left(x_{i}\right)\right)^{2}+\frac{\left(v_{A}\left(x_{i}\right)-v_{B}\left(x_{i}\right)\right)^{2}}{4 \pi^{2}}\right)\right)\right]^{1 / 2}, \\
D_{n E}(A, B) & =\left[\frac{1}{2 n}\left(\sum_{i=1}^{n}\left(\left(r_{A}\left(x_{i}\right)-r_{B}\left(x_{i}\right)\right)^{2}+\frac{\left(v_{A}\left(x_{i}\right)-v_{B}\left(x_{i}\right)\right)^{2}}{4 \pi^{2}}\right)\right)\right]^{1 / 2}, \\
D_{p}(A, B) & =\left[\frac{1}{2} \sum_{i=1}^{n}\left(\left|r_{A}\left(x_{i}\right)-r_{B}\left(x_{i}\right)\right|^{p}+\frac{\left(\min \left(\left|v_{A}\left(x_{i}\right)-v_{B}\left(x_{i}\right)\right|, 2 \pi-\left|v_{A}\left(x_{i}\right)-v_{B}\left(x_{i}\right)\right|\right)^{p}\right)}{\pi^{p}}\right)^{1 / p},\right. \\
D_{n p}(A, B) & =\left[\frac{1}{2 n} \sum_{i=1}^{n}\left(\left|r_{A}\left(x_{i}\right)-r_{B}\left(x_{i}\right)\right|^{p}+\frac{\left(\min \left(\left|v_{A}\left(x_{i}\right)-v_{B}\left(x_{i}\right)\right|, 2 \pi-\left|v_{A}\left(x_{i}\right)-v_{B}\left(x_{i}\right)\right|\right)^{p}\right)}{\pi^{p}}\right),\right.
\end{aligned}
$$


where $v_{A}(x), v_{B}(x) \in[0,2 \pi)$ for all $x \in X$.

Now, we give a brief summary of some properties of these distance measures for CFSs, as summarized in Table 1, in which $\sqrt{ }$ and $\times$, respectively, represent the corresponding invariance property holds or not.

Obviously, the primary difference between $D_{\mathrm{CFS}}(A, B)$ and other distance measures is that it is ratio scale invariant, but others are not.

Example 1. Let $X=\left\{x_{1}, x_{2}, x_{3}\right\}$; two CFSs $A$ and $B$ in $X$ are given as follows:

$$
\begin{aligned}
& A=\frac{0.6 e^{j \pi / 2}}{x_{1}}+\frac{1 e^{j 2 \pi / 3}}{x_{2}}+\frac{0.8 e^{j \pi / 2}}{x_{3}}, \\
& B=\frac{0.8 e^{j 3 \pi / 2}}{x_{1}}+\frac{0.2 e^{j \pi}}{x_{2}}+\frac{0.4 e^{j \pi}}{x_{3}}
\end{aligned}
$$

Let $t=1 / 2$; then,

$$
\begin{aligned}
& t A=\frac{0.3 e^{j \pi / 2}}{x_{1}}+\frac{0.5 e^{j 2 \pi / 3}}{x_{2}}+\frac{0.4 e^{j \pi / 2}}{x_{3}}, \\
& t B=\frac{0.4 e^{j 3 \pi / 2}}{x_{1}}+\frac{0.1 e^{j \pi}}{x_{2}}+\frac{0.2 e^{j \pi}}{x_{3}} .
\end{aligned}
$$

Consider the case of $p=1$; we have

$$
\begin{aligned}
D_{\text {CFS }}(A, B) & =\frac{\pi}{3}, \\
D_{\text {CFS }}(t A, t B) & =\frac{\pi}{3}, \\
D_{Z}(A, B) & =0.8, \\
D_{Z}(t A, t B) & =0.5, \\
D_{H}(A, B) & =\frac{139}{120}, \\
D_{H}(t A, t B) & =\frac{97}{120}, \\
D_{n H}(A, B) & =\frac{139}{360}, \\
D_{n H}(t A, t B) & =\frac{97}{360}, \\
D_{E}(A, B) & \approx 0.7682, \\
D_{E}(t A, t B) & \approx 0.5245, \\
D_{n E}(A, B) & \approx 0.4435, \\
D_{n E}(t A, t B) & \approx 0.3028, \\
D_{p}(A, B) & =\frac{67}{30}, \\
D_{p}(t A, t B) & =\frac{23}{15}, \\
D_{n P}(A, B) & =\frac{67}{90}, \\
D_{n p}(t A, t B) & =\frac{23}{45} .
\end{aligned}
$$

Thus, $D_{\mathrm{CFS}}(A, B)=D_{\mathrm{CFS}}(t A, t B)$ and $D_{\circ}(A, B) \neq D_{\mathrm{o}}(t A$, $t B$ ) where ${ }^{\circ} \in\{\mathrm{Z}, \mathrm{H}, \mathrm{nH}, \mathrm{E}, \mathrm{nE}, \mathrm{p}, \mathrm{np}\}$.

\section{Robustness of Complex Fuzzy Connectives and Complex Fuzzy Inference}

Now we consider the problem, if there is a small variance of the phase term of inputs, how much might the phase term of output vary?

\subsection{Robustness of Complex Fuzzy Connectives}

Lemma 1 (see [40]). Suppose $f, g$ are two bounded, real valued functions in $X$, then

$$
\begin{aligned}
& \left|\bigvee_{x \in X} f(x)-\bigvee_{x \in X} g(x)\right| \leq \bigvee_{x \in X}|f(x)-g(x)| \\
& \left|\bigvee_{x \in X} f(x)-\bigvee_{x \in X} g(x)\right| \leq \bigvee_{x \in X}|f(x)-g(x)|
\end{aligned}
$$

Theorem 7. Suppose $A, B$ are two CFSs in $X$, then

$$
\begin{aligned}
& C_{\mathrm{CFS}}\left(\Gamma_{1} A, \Gamma_{1} B\right)=C_{\mathrm{CFS}}(A, B), \\
& C_{\mathrm{CFS}}\left(\Gamma_{2} A, \Gamma_{2} B\right)=C_{\mathrm{CFS}}(A, B), \\
& C_{\mathrm{CFS}}\left(\Gamma_{3} A, \Gamma_{3} B\right)=C_{\mathrm{CFS}}(A, B) .
\end{aligned}
$$

Proof. Trivial.

Example 2. Let $X=\left\{x_{1}, x_{2}, x_{3}\right\}$; two CFSs $A$ and $B$ in $X$ are given as follows:

$$
\begin{aligned}
& A=\frac{0.4 e^{j 0.2 \pi}}{x_{1}}+\frac{0.2 e^{j 0.2 \pi}}{x_{2}}+\frac{0.7 e^{j 0.6 \pi}}{x_{3}}, \\
& B=\frac{0.5 e^{j 0.4 \pi}}{x_{1}}+\frac{0.3 e^{j 0.2 \pi}}{x_{2}}+\frac{0.6 e^{j 0.6 \pi}}{x_{3}} .
\end{aligned}
$$

Clearly, we have

$$
\begin{aligned}
& \Gamma_{1} A=\frac{0.6 e^{-j 0.2 \pi}}{x_{1}}+\frac{0.8 e^{-j 0.2 \pi}}{x_{2}}+\frac{0.3 e^{-j 0.6 \pi}}{x_{3}}, \\
& \Gamma_{1} B=\frac{0.5 e^{-j 0.4 \pi}}{x_{1}}+\frac{0.7 e^{-j 0.2 \pi}}{x_{2}}+\frac{0.4 e^{-j 0.6 \pi}}{x_{3}}, \\
& \Gamma_{2} A=\frac{0.6 e^{j 0.2 \pi}}{x_{1}}+\frac{0.8 e^{j 0.2 \pi}}{x_{2}}+\frac{0.3 e^{j 0.6 \pi}}{x_{3}}, \\
& \Gamma_{2} B=\frac{0.5 e^{j 0.4 \pi}}{x_{1}}+\frac{0.7 e^{j 0.2 \pi}}{x_{2}}+\frac{0.4 e^{j 0.6 \pi}}{x_{3}}, \\
& \Gamma_{3} A=\frac{0.6 e^{j 1.2 \pi}}{x_{1}}+\frac{0.8 e^{j 1.2 \pi}}{x_{2}}+\frac{0.3 e^{j 1.6 \pi}}{x_{3}}, \\
& \Gamma_{3} B=\frac{0.5 e^{j 1.4 \pi}}{x_{1}}+\frac{0.7 e^{j 1.2 \pi}}{x_{2}}+\frac{0.4 e^{j 1.6 \pi}}{x_{3}} .
\end{aligned}
$$

It is easy to verify that $C_{\mathrm{CFS}}\left(\Gamma_{1} A, \Gamma_{1} B\right)=C_{\mathrm{CFS}}(A, B)$, $C_{\mathrm{CFS}}\left(\Gamma_{2} A, \Gamma_{2} B\right)=C_{\mathrm{CFS}}(A, B)$, and $C_{\mathrm{CFS}}\left(\Gamma_{3} A, \Gamma_{3} B\right)=C_{\mathrm{CFS}}(A, B)$. 
TABle 1: Properties of distance measures for CFSs.

\begin{tabular}{lcccc}
\hline & Range & Reflectional invariance & Rotational invariance & Ratio scale invariance \\
\hline$D_{\text {CFS }}(A, B)$ & {$[0, \pi]$} & $\sqrt{ }$ & $\sqrt{ }$ & $\sqrt{ }$ \\
$D_{Z}(A, B)$ & {$[0,1]$} & $\sqrt{ }$ & $\times$ & $\times$ \\
$D_{H}(A, B)$ & {$[0, n]$} & $\sqrt{ }$ & $\times$ & $\times$ \\
$D_{n H}(A, B)$ & {$[0,1]$} & $\sqrt{ }$ & $\times$ & $\times$ \\
$D_{E}(A, B)$ & {$[0, \sqrt{n}]$} & $\sqrt{ }$ & $\times$ & $\times$ \\
$D_{n E}(A, B)$ & {$[0,1]$} & $\sqrt{ }$ & $\sqrt{ }$ & $\times$ \\
$D_{p}(A, B)$ & {$\left[0, n^{1 / p}\right]$} & $\sqrt{ }$ & $\sqrt{ }$ \\
$D_{n p}(A, B)$ & {$[0,1]$} & $\sqrt{ }$ & $\times$ \\
\hline
\end{tabular}

Theorem 8. Let $\epsilon_{1}, \epsilon_{2} \in[0, \pi / 4]$ and $A, B, C$ be CFSs in $X$; if Proof. Since we have $\vee_{x \in X}\left|v_{A}(x)-v_{B}(x)\right| \leq \epsilon_{1}$ and $\vee_{x \in X}\left|v_{B}(x)-v_{C}(x)\right|$ $\leq \epsilon_{2}$, then

$$
C_{\mathrm{CPS}}(A, C) \geq \cos \left(\epsilon_{1}+\epsilon_{2}\right) \text {. }
$$

$$
\bigvee_{x \in X}\left|v_{A}(x)-v_{C}(x)\right| \leq \bigvee_{x \in X}\left|v_{A}(x)-v_{B}(x)+v_{B}(x)-v_{C}(x)\right| \leq \bigvee_{x \in X}\left(\left|\nu_{A}(x)-v_{B}(x)\right|+\left|\nu_{B}(x)-v_{C}(x)\right|\right) \leq \epsilon_{1}+\epsilon_{2}
$$

then we have

$$
\begin{aligned}
C_{\mathrm{CFS}}(A, C) & \geq \bigvee_{i=1}^{n} \cos \left(\nu_{A}(x)-\nu_{C}(x)\right) \geq \bigvee_{i=1}^{n} \cos \left(\epsilon_{1}+\epsilon_{2}\right) \\
& \geq \cos \left(\epsilon_{1}+\epsilon_{2}\right)
\end{aligned}
$$

Example 3. Let $X=\left\{x_{1}, x_{2}, x_{3}\right\}$; two CFSs $A$ and $B$ in $X$ are given as follows:

$$
\begin{aligned}
& A=\frac{0.7 e^{j 0.52 \pi}}{x_{1}}+\frac{0.5 e^{j 0.22 \pi}}{x_{2}}+\frac{0.9 e^{j 0.61 \pi}}{x_{3}}, \\
& B=\frac{0.7 e^{j 0.54 \pi}}{x_{1}}+\frac{0.5 e^{j 0.23 \pi}}{x_{2}}+\frac{0.7 e^{j 0.62 \pi}}{x_{3}}, \\
& C=\frac{0.9 e^{j 0.63 \pi}}{x_{1}}+\frac{0.2 e^{j 0.25 \pi}}{x_{2}}+\frac{0.6 e^{j 0.55 \pi}}{x_{3}} .
\end{aligned}
$$

Clearly, we have $\vee_{x \in X}\left|\nu_{A}(x)-\nu_{B}(x)\right| \leq 0.02 \pi$ and $\vee_{x \in X}\left|\nu_{B}(x)-v_{C}(x)\right| \leq \epsilon_{1} \leq 0.09 \pi$. Thus, we can verify that $C_{\mathrm{CFS}}(A, C)=\max \cos (0.11 \pi), \cos (0.03 \pi), \cos (0.06 \pi)$ $\geq \max (\cos (0.11 \pi), \cos (0.11 \pi), \cos (0.11 \pi))$ $=\cos (0.11 \pi)=\cos (0.02 \pi+0.09 \pi)$.

Theorem 9. Let $\epsilon_{1}, \epsilon_{2} \in[0, \pi / 4]$ and $A, B, C, D$ be CFSs in $X$; if we have $\vee_{x \in X}\left|\nu_{A}(x)-v_{B}(x)\right| \leq \epsilon_{1}$ and $\vee_{x \in X} \mid v_{B}(x)-$ $v_{C}(x) \mid \leq \epsilon_{2}$, then

$$
\begin{aligned}
& C_{\mathrm{CFS}}(A \cup C, B \cup D) \geq \min \left(\cos \left(\epsilon_{1}\right), \cos \left(\epsilon_{2}\right)\right), \\
& C_{\mathrm{CFS}}(A \cap C, B \cap D) \geq \min \left(\cos \left(\epsilon_{1}\right), \cos \left(\epsilon_{2}\right)\right),
\end{aligned}
$$

where $\otimes \in\{\wedge, \vee\}$.

Proof. Let $\otimes=\vee$; from Lemma 1, we have

$$
v_{A}(x) \vee v_{C}(x)-v_{B}(x) \vee_{v D}(x) \leq \max \left(\left|v_{A}(x)-v_{B}(x)\right|,\left|v_{C}(x)-{ }_{v D}(x)\right|\right) \leq \max \left(\epsilon_{1}, \epsilon_{2}\right)
$$

Then, we have

$$
\begin{aligned}
C_{\mathrm{CFS}}(A \cup C, B \cup D) & \geq \bigvee_{i=1}^{n} \cos \left(v_{A \cup C}(x)-v_{B \cup D}(x)\right) \\
& \geq \bigvee_{i=1}^{n} \min \left(\cos \left(\epsilon_{1}\right), \cos \left(\epsilon_{2}\right)\right) \\
& \geq \min \left(\cos \left(\epsilon_{1}\right), \cos \left(\epsilon_{2}\right)\right) .
\end{aligned}
$$

The other cases can be similarly proved. 
Corollary 1. Suppose that $\epsilon_{i} \in[0, \pi / 4]$ and $\vee_{x \in X} \mid \nu_{A_{i}}$ $(x)-v_{B_{i}}(x) \mid \leq \epsilon_{i}(i=1,2, \ldots, n)$. For $\otimes \in\{\vee, \wedge\}$, we have

(i) $C_{C F S}\left(\bigcup_{i=1}^{n} A_{i}, \bigcup_{i=1}^{n} B_{i}\right) \geq \min _{i} \cos \left(\varepsilon_{i}\right)$

(ii) $C_{C F S}\left(\bigcap_{i=1}^{n} A_{i}, \bigcap_{i=1}^{n} B_{i}\right) \geq \min _{i} \cos \left(\epsilon_{i}\right)$

Corollary 2. Suppose that $\epsilon_{i j} \in[0, \pi / 4]$ and $\vee_{x \in X} \mid \nu_{A_{i j}}(x)-$ $v_{B_{i j}}(x) \mid \leq \epsilon_{i}, \quad\left(i=1,2, \ldots, n_{1}, j=1,2, \ldots, n_{2}\right)$. For $\otimes \in\{\bigvee, \wedge\}$, we have

(i) $C_{C F S}\left(\cap_{i=1}^{n_{1}} \cup_{i=1}^{n} A_{i j}, \cap_{i=1}^{n_{1}} \cup_{i=1}^{n} B_{i j}\right) \geq \min _{i, j} \cos \left(\varepsilon_{i, j}\right)$

(ii) $C_{C F S}\left(\cup_{i=1}^{n} \cap_{i=1}^{n_{1}} A_{i j}, \cup_{i=1}^{n} \cap_{i=1}^{n_{1}} B_{i j}\right) \geq \min _{i, j}^{i, j} \cos \left(\varepsilon_{i, j}\right)$
Theorem 10. Let $\epsilon_{1}, \epsilon_{2} \in[0, \pi / 4]$ and $A, B, C, D$ be CFSs in $X$; if we have $\vee_{x \in X}\left|v_{A}(x)-v_{B}(x)\right| \leq \epsilon_{2}$ and $\vee_{x \in X}\left|v_{C}(x)-{ }_{\nu D}(x)\right| \leq \epsilon_{2}$, then

$$
\begin{aligned}
& C_{\mathrm{CFS}}(A \cup C, B \cup D) \geq \min \left(\cos \left(\epsilon_{1}\right), \cos \left(\epsilon_{2}\right)\right), \\
& C_{\mathrm{CFS}}(A \cap C, B \cap D) \geq \min \left(\cos \left(\epsilon_{1}\right), \cos \left(\epsilon_{2}\right)\right),
\end{aligned}
$$

where $\otimes \in\{+,-\}$.

Proof. Let $\otimes=+$; from Lemma 1, we have

$$
\nu_{A}(x)+\nu_{C}(x)-\nu_{B}(x)+{ }_{\nu D}(x) \leq\left|\nu_{A}(x)-\nu_{B}(x)\right|+\left|\nu_{C}(x)-{ }_{\nu D}(x)\right| \leq \varepsilon_{1}+\varepsilon_{2} .
$$

Then, we have

$$
\begin{aligned}
C_{\mathrm{CFS}}(A \cup C, B \cup D) & \geq \bigvee_{i=1}^{n} \cos \left(\nu_{A \cup C}(x)-\nu_{B \cup D}(x)\right) \\
& \geq \bigvee_{i=1}^{n}\left(\cos \left(\varepsilon_{1}\right)+\cos \left(\varepsilon_{2}\right)\right) \geq \cos \left(\varepsilon_{1}+\varepsilon_{2}\right)
\end{aligned}
$$

The other cases can be similarly proved.

Corollary 3. Suppose that $\epsilon_{1} \in[0, \pi / 4]$ and $\vee_{x \in X} \mid \nu_{A_{i}}$ $(x)-v_{B_{i}}(x) \mid \leq \epsilon_{i}(i=1,2, \ldots, n)$. For $\otimes \in\{+,-\}$, we have

(i) $C_{C F S}\left(\cup_{i=1}^{n} A_{i}, \cup_{i=1}^{n} B_{i}\right) \geq \cos \left(\sum_{i}^{n} \varepsilon_{i}\right)$

(ii) $C_{C F S}\left(\cap_{i=1}^{n} A_{i}, \cap_{i=1}^{n} B_{i}\right) \geq \cos \left(\sum_{i}^{n} \varepsilon_{i}\right)$

Corollary 4. Suppose that $\epsilon_{i j} \in\left[0, \pi / 4 n_{1} n_{2}\right]$ and $\vee_{x \in X}\left|v_{A_{i j}}(x)-v_{B_{i j}}(x)\right| \leq \epsilon_{i}, \quad\left(i=1,2, \ldots, n_{1}, j=1,2, \ldots\right.$, $\left.n_{2}\right)$. For $\otimes \in\{+,-\}$, we have

(i) $C_{C F S}\left(\cap_{i=1}^{n_{1}} \cup_{i=1}^{n} A_{i j}, \cap_{i=1}^{n_{1}} \cup_{i=1}^{n} B_{i j}\right) \geq \cos \left(\sum_{i, j} \varepsilon_{i, j}\right)$

(ii) $C_{C F S}\left(\cup_{i=1}^{n} \cap_{i=1}^{n_{1}} A_{i j}, \cup_{i=1}^{n} \cap_{i=1}^{n_{1}} B_{i j}\right) \geq \cos \left(\sum_{i, j} \varepsilon_{i, j}\right)$

Example 4. Let $X=\left\{x_{1}, x_{2}, x_{3}\right\}$; two CFSs $A$ and $B$ in $X$ are given as follows:

$$
\begin{aligned}
& A=\frac{0.6 e^{j 0.52 \pi}}{x_{1}}+\frac{0.1 e^{j 0.22 \pi}}{x_{2}}+\frac{0.8 e^{j 0.61 \pi}}{x_{3}}, \\
& B=\frac{0.5 e^{j 0.51 \pi}}{x_{1}}+\frac{0.3 e^{j 0.23 \pi}}{x_{2}}+\frac{0.8 e^{j 0.62 \pi}}{x_{3}}, \\
& C=\frac{0.8 e^{j 1.13 \pi}}{x_{1}}+\frac{0.2 e^{j 1.25 \pi}}{x_{2}}+\frac{0.4 e^{j 1.45 \pi}}{x_{3}}, \\
& D=\frac{0.8 e^{j 1.23 \pi}}{x_{1}}+\frac{0.3 e^{j 1.25 \pi}}{x_{2}}+\frac{0.4 e^{j 1.55 \pi}}{x_{3}} .
\end{aligned}
$$

Clearly, we have $\vee_{x \in X}\left|\nu_{A}(x)-v_{B}(x)\right| \leq 0.01 \pi$ and $\vee_{x \in X}\left|\nu_{C}(x)-{ }_{v D}(x)\right| \leq 0.1 \pi$. Let $\star$ be the min t-norm, $\otimes=+$; then,

$$
\begin{aligned}
& A \cap C=\frac{0.6 e^{j 1.65 \pi}}{x_{1}}+\frac{0.1 e^{j 1.47 \pi}}{x_{2}}+\frac{0.4 e^{j 2.06 \pi}}{x_{3}}, \\
& B \cap D=\frac{0.5 e^{j 1.74 \pi}}{x_{1}}+\frac{0.3 e^{j 1.48 \pi}}{x_{2}}+\frac{0.4 e^{j 2.17 \pi}}{x_{3}} .
\end{aligned}
$$

Thus, we can verify that

$$
\begin{aligned}
C_{\mathrm{CFS}}(A, B) & =\max (\cos (0.09 \pi), \cos (0.01 \pi), \cos (0.11 \pi)) \\
& =\cos (0.11 \pi)=\cos (0.01 \pi+0.1 \pi) .
\end{aligned}
$$

Let $\star$ be the min t-norm, $\otimes=\wedge$; then,

$$
\begin{aligned}
& A \cap C=\frac{0.6 e^{j 0.52 \pi}}{x_{1}}+\frac{0.1 e^{j 0.22 \pi}}{x_{2}}+\frac{0.4 e^{j 0.61 \pi}}{x_{3}}, \\
& B \cap D=\frac{0.5 e^{j 0.51 \pi}}{x_{1}}+\frac{0.3 e^{j 0.23 \pi}}{x_{2}}+\frac{0.4 e^{j 0.62 \pi}}{x_{3}} .
\end{aligned}
$$

Thus, we can verify that

$$
\begin{aligned}
C_{\mathrm{CFS}}(A, B) & =\max (\cos (0.01 \pi), \cos (0.01 \pi), \cos (0.01 \pi)) \\
& =\cos (0.01 \pi)=\min (\cos (0.01 \pi), \cos (0.01 \pi)) .
\end{aligned}
$$

5.2. Robustness of Ramot et al.'s Complex Fuzzy Inference Method. Now, we study the robustness of Ramot et al.'s [15] complex fuzzy inference method. We only consider fuzzy modus ponens (FMP) of complex fuzzy inference, i.e., given an input CFS $A^{*}$ and a complex fuzzy rule $A \longrightarrow B$; then, infer an output CFS $B^{*}$, where $A, A^{*}$ are CFSs on $X$ and $B, B^{*}$ are CFSs on $Y$.

In [15], $A \longrightarrow B$ is represented by a complex fuzzy relation $R(X, Y)$, and the output $B^{*}$ is denoted by $\operatorname{FMP}\left(R, A^{*}\right)$. 
Then, $B^{*}$ is obtained as follows:

$$
\begin{aligned}
& r_{B^{*}}(y)=\sup _{x \in X}\left[r_{A^{*}}(x) \star r_{R}(x, y)\right], \\
& v_{B^{*}}(y)=g\left(v_{A^{*}}(x) \otimes v_{R}(x, y)\right),
\end{aligned}
$$

where $\otimes \in\{+,-, \vee, \wedge\}$ and $g$ is one of the following functions:

$$
\begin{gathered}
v_{B^{*}}(y)=\sup _{x \in X}\left(v_{A^{*}}(x) \otimes v_{R}(x, y)\right), \\
v_{B^{*}}(y)=\inf _{x \in X}\left(\nu_{A^{*}}(x) \otimes v_{R}(x, y)\right), \\
v_{B^{*}}(y)=\sum_{x \in X}\left(v_{A^{*}}(x) \otimes v_{R}(x, y)\right) .
\end{gathered}
$$

Theorem 11. Let $\epsilon \in[0, \pi / 4], g$ be the function of (54) or (55), and $\otimes \in\{\vee, \wedge\}$; if $\vee_{x \in X}\left|\nu_{A^{*}}(x)-\nu_{A *^{\prime}}(x)\right| \leq \varepsilon$, then

$$
C_{\mathrm{CFS}}\left(\operatorname{FMP}\left(R, A^{*}\right), \operatorname{FMP}\left(R,{ }^{A *^{\prime}}\right)\right) \geq \cos (\epsilon) .
$$

Proof. Trivial from Corollaries 1 and 2.

Theorem 12. Let $|X|=n, \epsilon \in[0, \pi / 4 n], g$ be the function of (56), and $\otimes \in\{+,-\}$; if $\vee_{x \in X}\left|\nu_{A^{*}}(x)-v_{A *^{\prime}}(x)\right| \leq \varepsilon$, then

$$
C_{\mathrm{CFS}}\left(\operatorname{FMP}\left(R, A^{*}\right), \operatorname{FMP}\left(R,{ }^{A *^{\prime}}\right)\right) \geq \cos (n \epsilon) .
$$

Proof. Trivial from Corollaries 3 and 4.

Example 6. Let $X=\left\{x_{1}, x_{2}, x_{3}\right\}$ and $Y=\left\{y_{1}, y_{2}, y_{3}\right\}$; a complex fuzzy relation $R(X, Y)$ on $X \times Y$ is given as follows:

$$
R(X, Y)=\left[\begin{array}{lll}
e^{j 0.5 \pi} & e^{j 0.2 \pi} & e^{j 0.3 \pi} \\
e^{j 0.6 \pi} & e^{j 0.7 \pi} & e^{j 0.3 \pi} \\
e^{j 0.7 \pi} & e^{j 0.4 \pi} & e^{j 0.4 \pi}
\end{array}\right]
$$

Two CFSs are, respectively, given as follows:

$$
\begin{aligned}
A^{*} & =\frac{e^{j 1.1 \pi}}{x_{1}}+\frac{e^{j 0.3 \pi}}{x_{2}}+\frac{e^{j 1.0 \pi}}{x_{3}} \\
A *^{\prime} & =\frac{e^{j 1.2 \pi}}{x_{1}}+\frac{e^{j 0.4 \pi}}{x_{2}}+\frac{e^{j 1.1 \pi}}{x_{3}} .
\end{aligned}
$$

Clearly, we have $\vee_{x \in X}\left|v_{A^{*}}(x)-\nu_{A *^{\prime}}(x)\right| \leq 0.1 \pi$. Let $g$ be the function of $(55)$ and $\otimes=\wedge$; then,

$$
\begin{aligned}
& \operatorname{FMP}\left(R, A^{*}\right)=\frac{e^{j 0.3 \pi}}{y_{1}}+\frac{e^{j 0.2 \pi}}{y_{2}}+\frac{e^{j 0.3 \pi}}{y_{3}} \\
& \operatorname{FMP}\left(R, A^{*}\right)=\frac{e^{j 0.4 \pi}}{y_{1}}+\frac{e^{j 0.2 \pi}}{y_{2}}+\frac{e^{j 0.2 \pi}}{y_{3}} .
\end{aligned}
$$

Thus, we can verify that

$$
C_{\mathrm{CFS}}\left(\operatorname{FMP}\left(R, A^{*}\right), \operatorname{FMP}\left(R,{ }^{A *^{\prime}}\right)\right)=\max (\cos (0.1 \pi), \cos (0), \cos (0.1 \pi))=\cos (0.1 \pi)
$$

Let $g$ be the function of $(56)$ and $\otimes=+$; then,

$$
\begin{aligned}
\operatorname{FMP}\left(R, A^{*}\right) & =\frac{e^{j 4.2 \pi}}{y_{1}}+\frac{e^{j 3.7 \pi}}{y_{2}}+\frac{e^{j 3.4 \pi}}{y_{3}}, \\
\operatorname{FMP}\left(R,{ }^{A *^{\prime}}\right) & =\frac{e j 4.5 \pi}{y_{1}}+\frac{e^{j 3.9 \pi}}{y_{2}}+\frac{e^{j 3.7 \pi}}{y_{3}} . \\
C_{\mathrm{CFS}}\left(\operatorname{FMP}\left(R, A^{*}\right), \operatorname{FMP}\left(R, A^{*}\right)\right) & =\max (\cos (0.3 \pi), \cos (0.2 \pi), \cos (0.3 \pi)) \\
& =\cos (0.3 \pi)=\cos (3 \times 0.1 \pi) .
\end{aligned}
$$

Thus, we can verify that

\section{Conclusion}

In this paper, a cosine similarity measure was proposed for CFSs by considering CFSs as sets of vectors in complex unit disk. In particular, the proposed cosine similarity measure is rotationally invariant, reflectionally invariant, and ratio scale invariant. All the existing measures of CFS in [40-42] are not ratio scale invariant. Finally, we applied the proposed similarity to measure the robustness of complex fuzzy connectives and Ramot et al.'s complex fuzzy inference.
We should note that the similarity measure presented in this paper mostly depends on the phase term of CFSs. Our robustness results for complex fuzzy connectives are estimated based on the perturbation of the phase term of CFSs. It may be a little extreme. So, how to apply to these results in the real word is another problem of interest. In [43], Ma et al. proposed a CFS-based prediction method which can handle the uncertainty and periodicity simultaneously, in which the modulus part is used to describe the semantic uncertainty feature and the phase part is for the temporal periodicity 
feature. In [21, 22], Bi et al. studied target selection application of CFSs, in which the modulus part is used to describe the distance and the phase part is for the direction of the target. Therefore, it will be meaningful to further investigate the real world application of these approaches with periodic (or direction) perturbations.

In the future, the proposed similarity measure can be extended to different complex fuzzy environments such as complex intuitionistic fuzzy set [44], complex Pythagorean fuzzy set [45], complex neutrosophic set [46], and complex q-rung orthopair fuzzy set [47] environments.

\section{Data Availability}

The data used to support the findings of this study are included in the article.

\section{Conflicts of Interest}

The authors declare that they have no conflicts of interest.

\section{Acknowledgments}

This research was funded by the Key Projects of Zhejiang Province's Educational Science Planning (2017SB068) and Humanities and Social Science Project of Chinese Ministry of Education (20YJAZH033).

\section{References}

[1] H. Bustince, E. Barrenechea, and M. Pagola, "Image thresholding using restricted equivalence functions and maximizing the measures of similarity," Fuzzy Sets and Systems, vol. 158, no. 5, pp. 496-516, 2007.

[2] H. Bustince, E. Barrenechea, and M. Pagola, "Relationship between restricted dissimilarity functions, restricted equivalence functions and normal EN-functions: image thresholding invariant," Pattern Recognition Letters, vol. 29, no. 4, pp. 525-536, 2008.

[3] S. H. Lee, W. Pedrycz, and G. Sohn, "Design of similarity and dissimilarity measures for fuzzy sets on the basis of distance measure," International Journal of Fuzzy System, vol. 11, no. 2, pp. 67-72, 2009.

[4] A. Bhattacharya, "On a measure of divergence of two multinomial populations," Sankhya, vol. 7, pp. 401-406, 1946.

[5] G. Salton and M. J. McGill, Introduction to Modern Information Retrieval, McGraw-Hill Book Company, New York, NY, USA, 1983.

[6] J. Ye, "Cosine similarity measures for intuitionistic fuzzy sets and their applications," Mathematical and Computer Modelling, vol. 53, no. 1-2, pp. 91-97, 2011.

[7] J. Ye, "Similarity measures of intuitionistic fuzzy sets based on cosine function for the decision making of mechanical design schemes," International Journal of Fuzzy System, vol. 30, no. 1, pp. 151-158, 2016.

[8] J. Ye, "Interval-valued intuitionistic fuzzy cosine similarity measures for multiple attribute decision-making," International Journal of General Systems, vol. 42, no. 8, pp. 883-891, 2013.

[9] G. Wei and Y. Wei, "Similarity measures of Pythagorean fuzzy sets based on the cosine function and their applications,"
International Journal of Intelligent Systems, vol. 33, no. 3, pp. 634-652, 2018.

[10] G. Wei, "Some cosine similarity measures for picture fuzzy sets and their applications to strategic decision making," Informatica, vol. 28, no. 3, pp. 547-564, 2017.

[11] L. L. Shi and J. Ye, "Study on fault diagnosis of turbine using an improved cosine similarity measure for vague sets," Journal of Applied Sciences, vol. 13, no. 10, pp. 1781-1786, 2013.

[12] J. Ye, "Vector similarity measures of hesitant fuzzy sets and their multiple attribute," Economic Computation \& Economic Cybernetics Studies \& Research, vol. 48, no. 4, pp. 206-217, 2014.

[13] J. Ye, "Vector similarity measures of simplified neutrosophic sets and their application in multicriteria decision making," International Journal of Fuzzy System, vol. 16, no. 2, pp. 204-211, 2014.

[14] D. Ramot, R. Milo, M. Friedman, and A. Kandel, "Complex fuzzy sets," IEEE Transactions on Fuzzy Systems, vol. 10, no. 2, pp. 171-186, 2002.

[15] D. Ramot, M. Friedman, G. Langholz, and A. Kandel, "Complex fuzzy logic," IEEE Transactions on Fuzzy Systems, vol. 11, no. 4, pp. 450-461, 2003.

[16] S. Dick, "Toward complex fuzzy logic," IEEE Transactions on Fuzzy Systems, vol. 13, no. 3, pp. 405-414, 2005.

[17] S. Dai, "A generalization of rotational invariance for complex fuzzy operations," IEEE Transactions on Fuzzy Systems .

[18] S. Dai, "Comment on toward complex fuzzy logic," IEEE Transactions on Fuzzy Systems, p. 1, 2019.

[19] S. Dai, "Complex fuzzy ordered weighted distance measures," Iranian Journal of Fuzzy Systems.

[20] L. Bi, Z. Zeng, B. Hu, and S. Dai, "Two classes of entropy measures for complex fuzzy sets," Mathematics, vol. 7, no. 1, p. 96, 2019.

[21] L. Bi, S. Dai, and B. Hu, "Complex fuzzy geometric aggregation operators," Symmetry, vol. 10, no. 7, p. 251, 2018.

[22] L. Bi, S. Dai, B. Hu, and S. Li, "Complex fuzzy arithmetic aggregation operators," International Journal of Fuzzy System, vol. 36, no. 3, pp. 2765-2771, 2019.

[23] S. Dai, L. Bi, and B. Hu, "Distance measures between the interval-valued complex fuzzy sets," Mathematics, vol. 7, no. 6, p. 549, 2019.

[24] S. Greenfield, F. Chiclana, and S. Dick, "Interval-valued complex fuzzy logic," in Proceedings of the 2016 IEEE International Conference on Fuzzy Systems (FUZZ-IEEE), pp. 2014-2019, IEEE, Vancouver, Canada, July 2016.

[25] S. Greenfield, F. Chiclana, and S. Dick, "Join and meet operations for interval-valued complex fuzzy logic," in Proceedings of the 2016 Annual Conference of the North American, IEEE, Lafayette, Louisiana, USA, pp. 1-5, November 2016.

[26] B. Hu, L. Bi, and S. Dai, "The orthogonality between complex fuzzy sets and its application to signal detection," Symmetry, vol. 9, no. 9, p. 175, 2017.

[27] L. Bi, B. Hu, S. Li, and S. Dai, "The parallelity of complex fuzzy sets and parallelity preserving operators," Journal of Intelligent \& Fuzzy Systems, vol. 34, no. 6, pp. 4173-4180, 2018.

[28] B. Hu, L. Bi, S. Dai, and S. Li, "The approximate parallelity of complex fuzzy sets," Journal of Intelligent \& Fuzzy Systems, vol. 35, no. 6, pp. 6343-6351, 2018.

[29] K. Y. Cai, "Robustness of fuzzy reasoning and $\delta$-equalities of fuzzy sets," IEEE Transactions on Fuzzy Systems, vol. 9, no. 5, pp. 738-750, 2001.

[30] M. S. Ying, "Perturbation of fuzzy reasoning," IEEE Transactions on Fuzzy Systems, vol. 7, no. 5, pp. 625-629, 1999. 
[31] Y. Li, K. Qin, and X. He, "Robustness of fuzzy connectives and fuzzy reasoning," Fuzzy Sets and Systems, vol. 225, pp. 93-105, 2013.

[32] Y. Li, K. Qin, X. He, and D. Meng, "Robustness of fuzzy connectives and fuzzy reasoning with respect to general divergence measures," Fuzzy Sets and Systems, vol. 294, pp. 63-78, 2016.

[33] Y. Li, D. Li, W. Pedrycz, and J. Wu, "An approach to measure the robustness of fuzzy reasoning," International Journal of Intelligent Systems, vol. 20, no. 4, pp. 393-413, 2005.

[34] S. Dai, D. Pei, and S.-m. Wang, "Perturbation of fuzzy sets and fuzzy reasoning based on normalized Minkowski distances," Fuzzy Sets and Systems, vol. 189, no. 1, pp. 63-73, 2012.

[35] S. Dai, D. Pei, and D. Guo, "Robustness analysis of full implication inference method," International Journal of Approximate Reasoning, vol. 54, no. 5, pp. 653-666, 2013.

[36] M. Luo and K. Zhang, "Robustness of full implication algorithms based on interval-valued fuzzy inference," International Journal of Approximate Reasoning, vol. 62, pp. 61-72, 2015.

[37] M. Luo, L. Wu, and L. Fu, "Robustness analysis of the intervalvalued fuzzy inference algorithms1," Journal of Intelligent \& Fuzzy Systems, vol. 38, no. 1, pp. 685-696, 2020.

[38] M. Luo, Z. Cheng, and J. Wu, "Robustness of interval-valued universal triple I algorithms1," Journal of Intelligent \& Fuzzy Systems, vol. 30, no. 3, pp. 1619-1628, 2016.

[39] M. Luo and X. Zhou, "Robustness of reverse triple I algorithms based on interval-valued fuzzy inference," International Journal of Approximate Reasoning, vol. 66, pp. 16-26, 2015.

[40] G. Zhang, T. S. Dillon, K.-Y. Cai, J. Ma, and J. Lu, "Operation properties," International Journal of Approximate Reasoning, vol. 50, pp. 1227-1249, 2009.

[41] A. U. M. Alkouriand and A. R. Salleh, "Linguistic variables, hedges and several distances on complex fuzzy sets," Journal of Intelligent \& Fuzzy Systems, vol. 26, pp. 2527-2535, 2014.

[42] B. Hu, L. Bi, S. Dai, and S. Li, "Distances of complex fuzzy sets and continuity of complex fuzzy operations," Journal of Intelligent \& Fuzzy Systems, vol. 35, pp. 2247-2255, 2018.

[43] J. Ma, G. Zhang, and J. Lu, "A method for multiple periodic factor prediction problems using complex fuzzy sets," IEEE Transactions on Fuzzy Systems, vol. 20, pp. 32-45, 2012.

[44] A. Alkouri and A. Salleh, "Complex intuitionistic fuzzy sets," Fundamental and Applied Sciences, vol. 1482, pp. 464-470, 2012.

[45] K. Ullah, T. Mahmood, Z. Ali et al., "On some distance measures of complex pythagorean fuzzy sets and their applications in pattern recognition," Complex and Intelligent Systems, vol. 6, pp. 15-27, 2020.

[46] M. Ali and F. Smarandache, "Complex neutrosophic set," Neural Computing and Applications, vol. 28, no. 7, pp. 1817-1834, 2017.

[47] P. Liu, Z. Ali, and T. Mahmood, "A method to multi-attribute group decision-making problem with complex q-rung orthopair linguistic information based on Heronian mean operators," International Journal of Computational Intelligence Systems, vol. 12, pp. 1465-1496, 2019. 